\title{
Structural stability, electronic structure and magnetic properties of the new hypothetical half-metallic ferromagnetic full-Heusler alloy CoNiMnSi
}

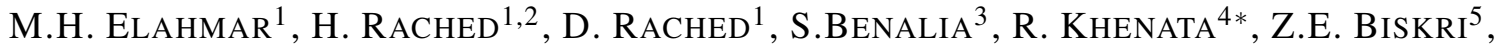 \\ S. BIN OMRAN ${ }^{6}$ \\ ${ }^{1}$ Laboratoire des Matériaux Magnétiques, Faculté des Sciences, Université Djillali Liabčs de Sidi Bel-Abbčs, \\ Sidi Bel-Abbčs 22000, Algérie \\ ${ }^{2}$ Université Hassiba Benbouali Chlef, Faculté des Sciences, Département de Physique, Chlef 02000, Algérie \\ ${ }^{3}$ Centre Universitaire de Tissemsilt, Tissemsilt 38000, Algérie \\ ${ }^{4}$ Laboratoire de Physique Quantique de Modélisation Mathématique (LPQ3M), Faculté des Sciences et Technologies, \\ Université de Mascara, Mascara 29000, Algérie \\ ${ }^{5}$ Unité de Recherche Sciences des Matériaux et Applications, Département de Physique, Faculté des Sciences Exactes, \\ Université Constantine 1, Constantine 25000, Algérie \\ ${ }^{6}$ Department of Physics and Astronomy, College of Science, King Saud University, P.O. Box 2455, \\ Riyadh 11451, Saudi Arabia
}

\begin{abstract}
We investigated the structural stability as well as the mechanical, electronic and magnetic properties of the Full-Heusler alloy CoNiMnSi using the full-potential linearized augmented plane wave (FP-LAPW) method. Two generalized gradient approximations (GGA and GGA $+\mathrm{U}$ ) were used to treat the exchange-correlation energy functional. The ground state properties of CoNiMnSi including the lattice parameter and bulk modulus were calculated. The elastic constants $\left(\mathrm{C}_{\mathrm{ij}}\right)$ and their related elastic moduli as well as the thermodynamic properties for CoNiMnSi have been calculated for the first time. The existence of half-metallic ferromagnetism (HM-FM) in this material is apparent from its band structure. Our results classify CoNiMnSi as a new HM-FM material with high spin polarization suitable for spintronic applications.
\end{abstract}

Keywords: ab-initio calculations; Heusler alloys; electronic structure; magnetic properties

(C) Wroclaw University of Technology.

\section{Introduction}

The first half-metallic ferromagnetic (HMF) material, the half-Heusler alloy NiMnSb, was discovered by de Groot using first-principles calculations [1]. In recent years, HMF materials have been intensively investigated because they exhibit metallic character for majority spin and semiconducting behavior for minority spin. Therefore, they are good candidates for spintronic applications, in which tunneling magnetoresistance (TMR) and giant magnetoresistance (GMR) are required [2]. In particular, Co-based Heusler alloys are ideal compounds for spintronic applications because

*E-mail: khenata_rabah@yahoo.fr they exhibit a high Curie temperature and a high spin polarization at the Fermi energy $\left(\mathrm{E}_{\mathrm{F}}\right)$.

Rai and coworkers [3], using the LSDA + $\mathrm{U}$ exchange correlation contribution, showed that $\mathrm{Co}_{2} \mathrm{FeGe}$ is a HMF. Candan and co-authors [4] used first-principles self-consistent pseudopotential plane wave calculations to study the magnetic moments, density of states and half-metallicity of L2 2 -type full-Heusler alloys with the empirical formula $\mathrm{Co}_{2} \mathrm{MnX}(\mathrm{X}=\mathrm{Si}, \mathrm{Ge}, \mathrm{Al}$ or $\mathrm{Ga})$. The aforementioned authors indicated that the $\mathrm{Co}_{2} \mathrm{MnAl}$, $\mathrm{Co}_{2} \mathrm{MnGa}, \mathrm{Co}_{2} \mathrm{MnSi}$ and $\mathrm{Co}_{2} \mathrm{MnGe}$ compounds all exhibit half-metallic (HM) character.

Full-Heusler alloys are intermetallic compounds with the stoichiometric composition $\mathrm{X}_{2} \mathrm{YZ}$, where $\mathrm{X}$ and $\mathrm{Y}$ are transition metal elements and 
$\mathrm{Z}$ is a III-, IV- or V-group element. These compounds crystallize in a cubic structure, as shown in Fig. 1. Full-Heusler compounds are found in either $\mathrm{Hg}_{2} \mathrm{CuTi}$-type, denoted X-type, or $\mathrm{Cu}_{2} \mathrm{MnAl}$ type, denoted $\mathrm{L} 2{ }_{1}$-type. When the valence electron number of the $\mathrm{X}$ element is higher than that of the $\mathrm{Y}$ element, the compound takes on the L21-type structure, while the X-type structure is favored in the reverse case. The unit cell of the L2 1 -type structure has the following occupied Wyckoff positions: $X_{1}=\left(\begin{array}{lll}0 & 0 & 0\end{array}\right), X_{2}=\left(\begin{array}{lll}1 / 2 & 1 / 2\end{array}\right.$ $1 / 2), Y=(1 / 41 / 41 / 4)$ and $Z=(3 / 43 / 43 / 4)$. For the $X$-type structure, in which the occupancy parameters of the $\mathrm{X}_{2}$ and $\mathrm{Y}$ elements are exchanged, the two $\mathrm{X}$ atoms are no longer equivalent, producing ternary and quaternary Full-Heusler alloys. Quaternary Heusler alloys with a stoichiometric composition of $\mathrm{XX}$ ' YZ generally crystallize as the LiMgPdSb-type Heusler structure, denoted Y-type, which belongs to the F43m space group [5, 6]. The unit cell for the Y-type structure has the following occupied Wyckoff positions: $X=(1 / 21 / 21 / 2)$, $\mathrm{X}^{\prime}=\left(\begin{array}{lll}0 & 0 & 0\end{array}\right), \mathrm{Y}=\left(\begin{array}{lll}1 / 4 & 1 / 4 & 1 / 4\end{array}\right), \mathrm{Z}=\left(\begin{array}{lll}1 / 4 & 1 / 4\end{array}\right.$ $1 / 4)$. Several recent studies regarding quaternary Heusler alloys have demonstrated that these compounds exhibit HMF [7-10]. Considerable theoretical work has been performed on quaternary Heusler compounds, particularly Co-based compounds. For example, a study carried out by Gao et al. [11] showed that all of the investigated alloys were excellent HM ferromagnets with large HM gaps. Motivated by the previous studies, our main purpose in this work was to carry out a computational investigation based on first-principles calculations focusing on the structural, mechanical, electronic and magnetic properties of the new hypothetical quaternary CoNiMnSi Heusler alloy. The primary objective was to observe a new instance of HMF in full-Heusler alloys. Our calculation was performed using the full-potential linearized augmented plane wave (FP-LAPW) method, as implemented in the WIEN2k code, in the framework of density functional theory (DFT) with the GGA and GGA + U approximations. To our knowledge, no theoretical or experimental work on the electronic states, spin polarization or magnetic properties of this Heusler alloy has been performed.

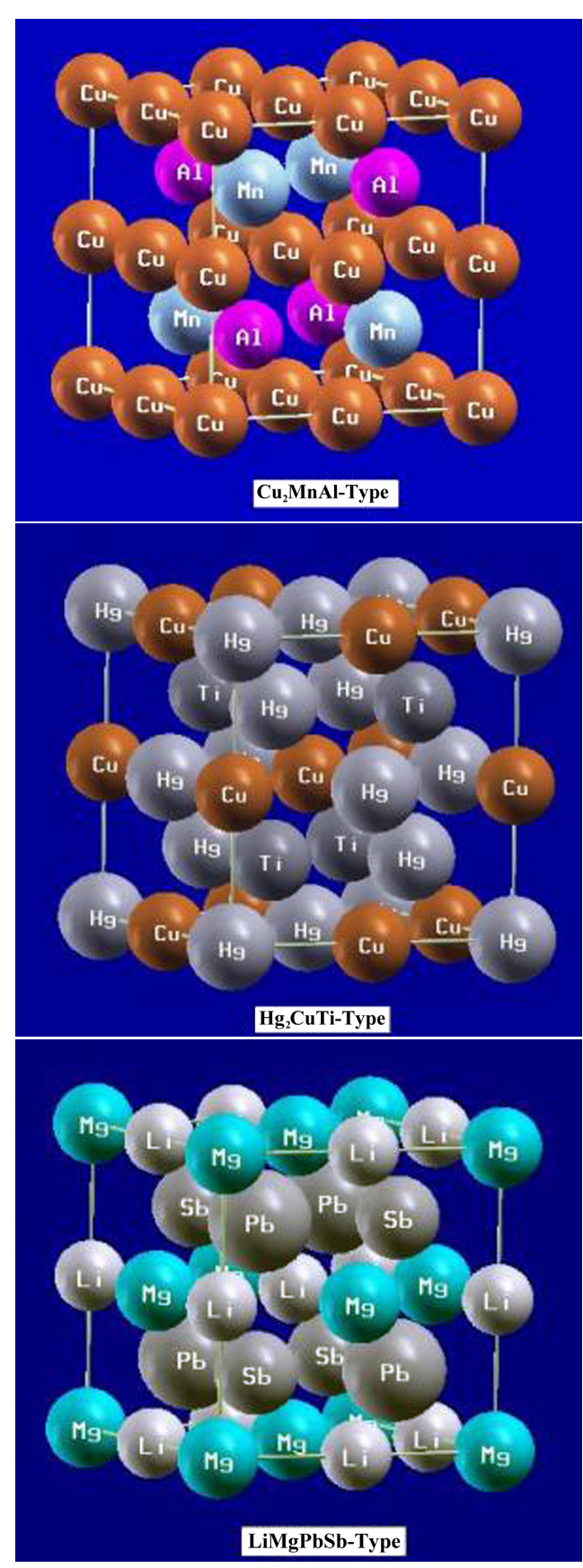

Fig. 1. Three crystalline structures of CoNiMnSi.

\section{Calculation method}

The calculations reported in this work were performed using the full-potential linearized augmented plane wave (FP-LAPW) method [1214] within the framework of density-functional 
theory (DFT) $[15,16]$ and implemented in WIEN2k code [17]. The exchange-correlation (XC) potential was treated with Perdew-BurkeErnzerhof generalized gradient approximations (GGAPBE) [18, 19]. The parameter of the plane wave cut-off $\left(\mathrm{R}_{\mathrm{MT}} \cdot \mathrm{Kmax}\right)$, which controls the number of basis functions, was set to 9.0 to ensure sufficient accuracy in our computed results. The muffin-tin radii (MT) for $\mathrm{Co}, \mathrm{Ni}, \mathrm{Mn}$, and $\mathrm{Si}$ were $2.22,2.22,2.22$ and 2.08 (a.u.), respectively. Within the spheres, the charge density and potential were expanded in terms of crystal harmonics up to an angular momentum of $\mathrm{L}=10$, and a plane wave was used in the interstitial region. The parameter $G_{\max }$, which defines the magnitude of the largest vector in the charge density Fourier expansion, was chosen to be 14 . The Brillouin zone integration was performed using the Monkhorst-Pack method with $3000 \mathrm{k}$-points in the first Brillouin zone [20]. The cut-off energy, which defines the separation of valence and core states, was chosen to be $-6 \mathrm{Ry}$. We selected the charge convergence to be 0.0001 during self-consistency cycles. In this framework, the strong Coulomb repulsion between localized $\mathrm{f}$ states was treated by adding a Hubbardlike term $U$ to the effective potential, following the approach of Anisimov et al. [21, 22] and Loschen et al. [23]. The addition of this term in the calculation leads to an improved description of correlation effects. The $\mathrm{U}$ values were chosen to be $3 \mathrm{eV}$ for Co-3d and Mn-3d and $2 \mathrm{eV}$ for Ni-3d.

\section{Results and discussion}

\subsection{Structural and mechanical properties}

We first optimized the unit cell volume by calculating the total energy as a function of volume of the quaternary Heusler alloy in its three structural phases, namely the $\mathrm{Hg}_{2} \mathrm{CuTi}$-type, $\mathrm{Cu}_{2} \mathrm{MnAl}$-type and LiMgPdSb-type structures for the ferromagnetic states. The calculated total energies within the GGA approximation as a function of the volume are displayed in Fig. 2. From the figure, one can see that the LiMgPbSb-type structure is the most stable due to its lowest total energy. The calculated total energies were fitted to Murnaghan's equation of state [24] to obtain ground state properties, such as the equilibrium lattice constant, the bulk modulus B and its pressure derivative B'. The theoretically calculated ground state properties of this compound at zero pressure and ambient temperature for its different phases are given in Table 1. Because there are, to the best of our knowledge, no comparable studies of this compound in the literature, we compare our results to previously reported theoretical data on the $\mathrm{Co}_{2} \mathrm{MnSi}$ compound $[4,25,26]$. Based on the good agreement between the present calculated values and the theoretical results for $\mathrm{Co}_{2} \mathrm{MnSi}$ obtained from the literature, we believe that our calculations of the structural properties of the three structural phases are reliable, and we encourage future experimental work to corroborate our calculated results.

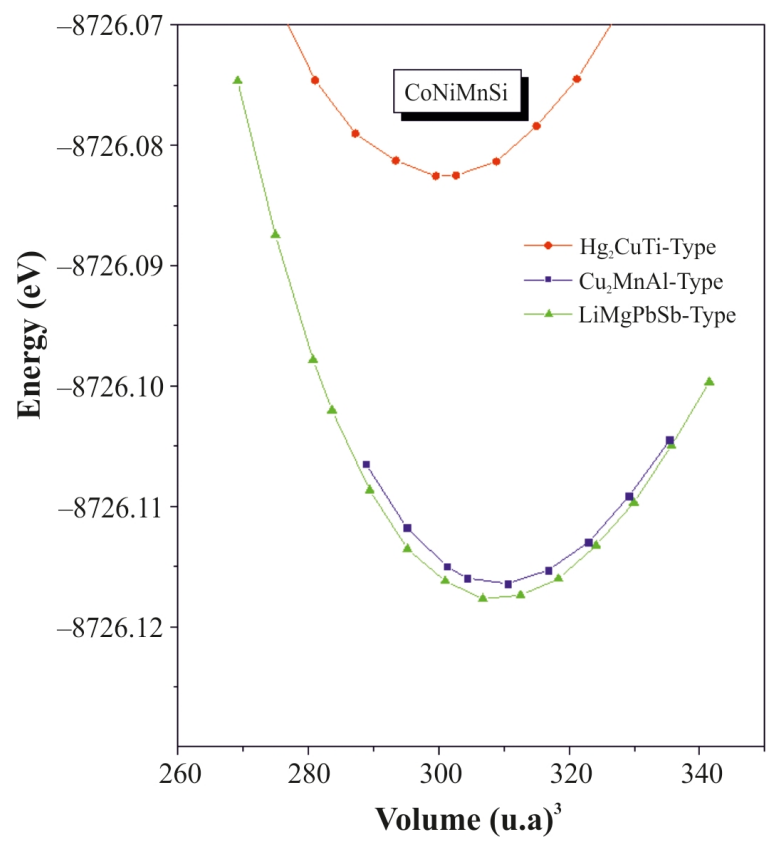

Fig. 2. Calculated total energy versus volume for CoNiMnSi.

We now turn our attention to discuss the mechanical properties of the CoNiMnSi compound. In particular, we will confirm its mechanical stability in the LiMgPbSb-type structure via calculation of the elastic constants $\mathrm{C}_{\mathrm{ij}}$. These constants are fundamental and indispensable for describing the mechanical properties of materials because they are closely related to various fundamental solid-state 
Table 1. Calculated equilibrium lattice parameters $\mathrm{a}_{0}(\AA ̊)$, bulk modulus $\mathrm{B}_{0}(\mathrm{GPa})$, its pressure derivative $\mathrm{B}_{0}^{\prime}(\mathrm{GPa})$ and equilibrium energy $\mathrm{E}_{0}$ (Ryd) for the CoNiMnSi compound in the different structures considered in this work.

\begin{tabular}{llllll}
\hline Material & Structure & $\mathrm{a}_{0}(\AA)$ & $\mathrm{B}_{0}(\mathrm{GPa})$ & $\mathrm{B}^{\prime}$ & $\mathrm{E}_{0}(\mathrm{Ryd})$ \\
\hline \hline CoNiMnSi & $\mathrm{Hg}_{2} \mathrm{CuTi}$-type & 5.6256 & 179.51 & 3.50 & -8726.0826 \\
& $\mathrm{Cu}_{2} \mathrm{MnAl}$-type & 5.6810 & 187.89 & 5.06 & -8726.1164 \\
& $\mathrm{LiMgPbSb-type}$ & 5.6787 & 187.6 & 4.81 & -8726.1177 \\
\hline $\mathrm{Co}_{2} \mathrm{MnSi}$ & $\mathrm{Cu}_{2} \mathrm{MnAl}$-type & $5.633^{\mathrm{a}}$ & $212.8^{\mathrm{a}}$ & $4.68^{\mathrm{a}}$ & - \\
& & $5.634^{b}$ & $226^{\mathrm{b}}$ & - & - \\
& & $5.639^{\mathrm{c}}$ & $214^{\mathrm{c}}$ & $4.674^{\mathrm{c}}$ & - \\
\hline
\end{tabular}

${ }^{\mathrm{a}}[4],{ }^{\mathrm{b}}[25],{ }^{\mathrm{c}}[26]$

phenomena, such as interatomic bonding, equations of state and phonon spectra. Elastic properties are also linked thermodynamically with specific heat, thermal expansion, Debye temperature, and the Grüneisen parameter. Most importantly, knowledge of the elastic constants is essential for many practical applications related to the mechanical properties of a solid. The elastic constants $\mathrm{C}_{\mathrm{ij}}$ are the proportionality coefficients relating the applied strain $\varepsilon_{\mathrm{i}}$ to the stress $\sigma_{\mathrm{i}}$, where $\sigma_{\mathrm{i}}=\mathrm{C}_{\mathrm{ij}} \varepsilon_{\mathrm{i}}$. Therefore, the $\mathrm{C}_{\mathrm{ij}}$ determine the response of the crystal to external forces. For a completely asymmetrical system, there are 21 independent elastic constants $\mathrm{C}_{\mathrm{ij}}$; however, the cubic symmetry of the studied crystal reduces this number to only three $\left(\mathrm{C}_{11}, \mathrm{C}_{12}\right.$ and $\mathrm{C}_{44}$ ) independent elastic constants. The elastic constants $\mathrm{C}_{\mathrm{ij}}$ are obtained by calculating the total energy as a function of volume, while conserving strains that break the symmetry. Further details of the calculation can be found in the literature [30]. The presence of mechanically stable phases or macroscopic stability depends on the positive definiteness of the stiffness matrix [31]. The following conditions are known as the Born Huang criteria [32], and they are defined as $\mathrm{C}_{11}>0, \mathrm{C}_{44}>0$, $\mathrm{C}_{11}>\mathrm{C}_{12}$ and $\left(\mathrm{C}_{11}+2 \mathrm{C}_{12}\right)>0$ for a cubic structure. The elastic constants $\mathrm{C}_{\mathrm{ij}}$ are estimated from first-principles calculations for a single crystal of our compound. However, the prepared materials are in general polycrystalline, therefore, it is important to evaluate the corresponding moduli for the polycrystalline species. For this purpose, we have derived other mechanical parameters, namely the shear modulus G, Young's modulus E and
Poisson's ratio $v$, which are the important elastic moduli for applications, using the following standard relations [33, 34]:

$$
\begin{gathered}
G=\frac{C_{11}-C_{12}+3 C_{44}}{5} \\
E=\frac{9 B G}{3 B+G} \\
v=\frac{3 B-2 G}{2(3 B+G)} \\
A=\frac{2 C_{44}}{C_{11}-C_{12}}
\end{gathered}
$$

Table 2 lists the elastic constants and elastic moduli for polycrystalline materials calculated within the GGA approximation. One can see that the LiMgPbSb-type structure for CoNiMnSi is mechanically stable at ambient conditions because the Born Huang criteria are satisfied. The $\mathrm{C}_{11}$ value, which is related to the unidirectional compression along the principal crystallographic direction, is much greater than that of $\mathrm{C}_{44}$, indicating a weak resistance to shear deformation compared with the resistance to unidirectional compression. We also note that this compound possesses a high bulk modulus and Young's modulus, what classifies this compound as a strong incompressible material. The typical value of Poisson's ratio $(v)$ for ionic materials is 0.25 [35]. The calculated value of Poisson's ratio for the studied compound is less than 
Table 2. Calculated single crystal elastic constants $\mathrm{C}_{\mathrm{ij}}(\mathrm{GPa})$, polycrystalline elastic modulus (GPa), shear modulus $\mathrm{G}(\mathrm{GPa})$, Young's modulus E (GPa), Poisson's ratio $v$ and shear anisotropic factor A for the CoNiMnSi compound.

\begin{tabular}{llllllll}
\hline Material & $\mathrm{C}_{11}$ & $\mathrm{C}_{12}$ & $\mathrm{C}_{44}$ & $\mathrm{G}$ & $\mathrm{E}$ & $v$ & $\mathrm{~A}$ \\
\hline \hline CoNiMnSi & 425.75 & 68.52 & 172.49 & 174.94 & 400.37 & 0.144 & 0.966 \\
\hline $\mathrm{Co}_{2} \mathrm{MnSi}$ & $311.16^{\mathrm{a}}$ & $164.75^{\mathrm{a}}$ & $153.26^{\mathrm{a}}$ & - & - & - & - \\
& $316^{\mathrm{b}, \mathrm{c}}$ & $174^{\mathrm{b}, \mathrm{c}}$ & $143^{\mathrm{b}, \mathrm{c}}$ & & & & \\
\hline
\end{tabular}

${ }^{\mathrm{a}}[4],{ }^{\mathrm{b}}[27],{ }^{\mathrm{c}}[28]$

0.25 , indicating a covalent contribution in the interatomic bonding. The calculated value of the shear anisotropic factor A for this compound obtained from GGA calculations is given in Table 2. The value of A must be one for an isotropic crystal, while any value smaller or greater than unity is a measure of the degree of elastic anisotropy possessed by the crystal [38]. The calculated value is slightly below unity, which means that this compound has a lower anisotropy and possesses a low probability to develop micro-cracks or structural defects during its growth process.

Once we have calculated the Young's modulus E, bulk modulus B and the shear modulus $G$, we may estimate the Debye temperature from the average sound velocity $v_{\mathrm{m}}[36]$ :

$$
\theta_{D}=\frac{h}{k_{B}}\left(\frac{3 n}{4 \pi V_{a}}\right)^{-\frac{1}{3}} v_{m}
$$

where $\mathrm{h}$ is Plank's constant, $\mathrm{k}_{\mathrm{B}}$ is Boltzmann's constant and $\mathrm{V}_{\mathrm{a}}$ is the atomic volume. The average sound velocity in the polycrystalline material is given by [36]:

$$
v_{m}=\left[\frac{1}{3}\left(\frac{2}{v_{t}^{3}}+\frac{1}{v_{l}^{3}}\right)\right]^{-\frac{1}{3}}
$$

where $v_{1}$ and $v_{t}$ are, respectively, the longitudinal and transverse sound velocities obtained using the shear modulus $G$ and the bulk modulus B from Navier's equation [37]:

$$
\begin{gathered}
v_{l}=\left(\frac{3 B+G}{3 \rho}\right)^{\frac{1}{2}} \\
v_{t}=\left(\frac{G}{\rho}\right)^{\frac{1}{2}}
\end{gathered}
$$

The calculated sound velocities and Debye temperature for the CoNiMnSi compound in the LiMgPbSb-type structure are listed in Table 3. To the best of our knowledge, the sound velocities, Debye temperature, elastic constants and their related properties have not yet been established for this compound. We hope that our calculations can be used to make up for the lack of data on this compound.

Table 3. Calculated longitudinal, transverse and average sound velocity $\left(\mathrm{v}_{\mathrm{l}}, \mathrm{v}_{\mathrm{t}}\right.$, and $\mathrm{v}_{\mathrm{m}}$, in $\mathrm{ms}^{-1}$ ) and Debye temperature $\left(\theta_{\mathrm{D}}, \mathrm{K}\right)$ for the CoNiMnSi compound.

\begin{tabular}{ccccc}
\hline $\begin{array}{c}\mathrm{T}=0) \text { Material } \\
(\mathrm{P}=0)\end{array}$ & $\begin{array}{c}\mathrm{V}_{\mathrm{S}} \\
{[\mathrm{m} / \mathrm{s}]}\end{array}$ & $\begin{array}{c}\mathrm{V}_{\mathrm{l}} \\
{[\mathrm{m} / \mathrm{s}]}\end{array}$ & $\begin{array}{c}\mathrm{V}_{\mathrm{m}} \\
{[\mathrm{m} / \mathrm{s}]}\end{array}$ & $\begin{array}{c}\theta_{\mathrm{D}} \\
{[\mathrm{K}]}\end{array}$ \\
\hline \hline CoNiMnSi & 4902.70 & 7604.25 & 5381.81 & 445.20 \\
\hline
\end{tabular}

\subsection{Electronic and magnetic properties}

In the following paragraph we shed more light on the electronic properties of the CoNiMnSi compound in its stable phase by calculating the total and atomic site-projected 1-decomposed densities of states (TDOS and PDOS) for the spin-up and spin-down electrons using the GGA and GGA + $\mathrm{U}$ approximations. The electronic valence states for the studied compound are $4 \mathrm{~s} / 4 \mathrm{p} / 3 \mathrm{~d}$ for $\mathrm{Co}, \mathrm{Ni}$ and $\mathrm{Mn}$ and $3 \mathrm{~s} / 3 \mathrm{p}$ for $\mathrm{Si}$. The plots of the TDOS and PDOS functions computed with both GGA and GGA + U are displayed in Fig. 3 and Fig. 4, respectively. One can see, no energy gap at the Fermi level for the minority spin states with the GGA approximation, indicating that this compound has ferromagnetism-false half-metallic character at the 
equilibrium state. However, the GGA + U approximation yielded a gap of $0.17 \mathrm{eV}$ in the minority spin, as shown in Fig. 4. This gap is due to the strong hybridization between the $\mathrm{Co} / \mathrm{Ni} / \mathrm{Mn}-\mathrm{d}$ states, thus, confirms that our compound exhibits half-metallic ferromagnetism. From a visual inspection, the TDOS spectrum is divided into two regions. The first one situated between $-5 \mathrm{eV}$ and $2.5 \mathrm{eV}$ is due to the strong hybridization of the $3 \mathrm{~d}-$ Co, $3 \mathrm{~d}-\mathrm{Ni}$ and $3 \mathrm{~d}-\mathrm{Mn}$ states. The second is the region outside of the energy range of -5 to $2.5 \mathrm{eV}$, which is dominated by Si-s,p states.

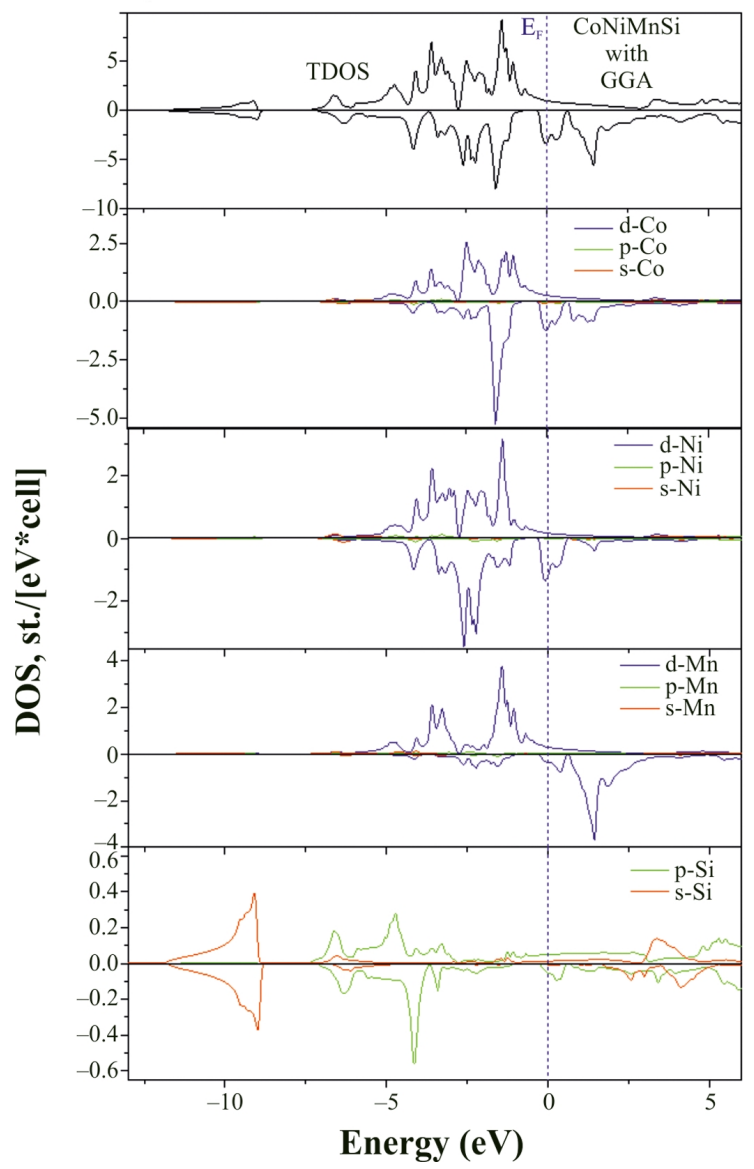

Fig. 3. Total and partial densities of states (TDOS, PDOS) for CoNiMnSi in its stable structure types calculated using the GGA approximation.

To understand the nature of the chemical bonding, we have also calculated the contours of the charge densities in the (lll $\left.\begin{array}{lll}1 & 1 & 0\end{array}\right)$ plane with both the GGA and GGA + U approximations, and the results are displayed in Fig. 5 and Fig. 6,

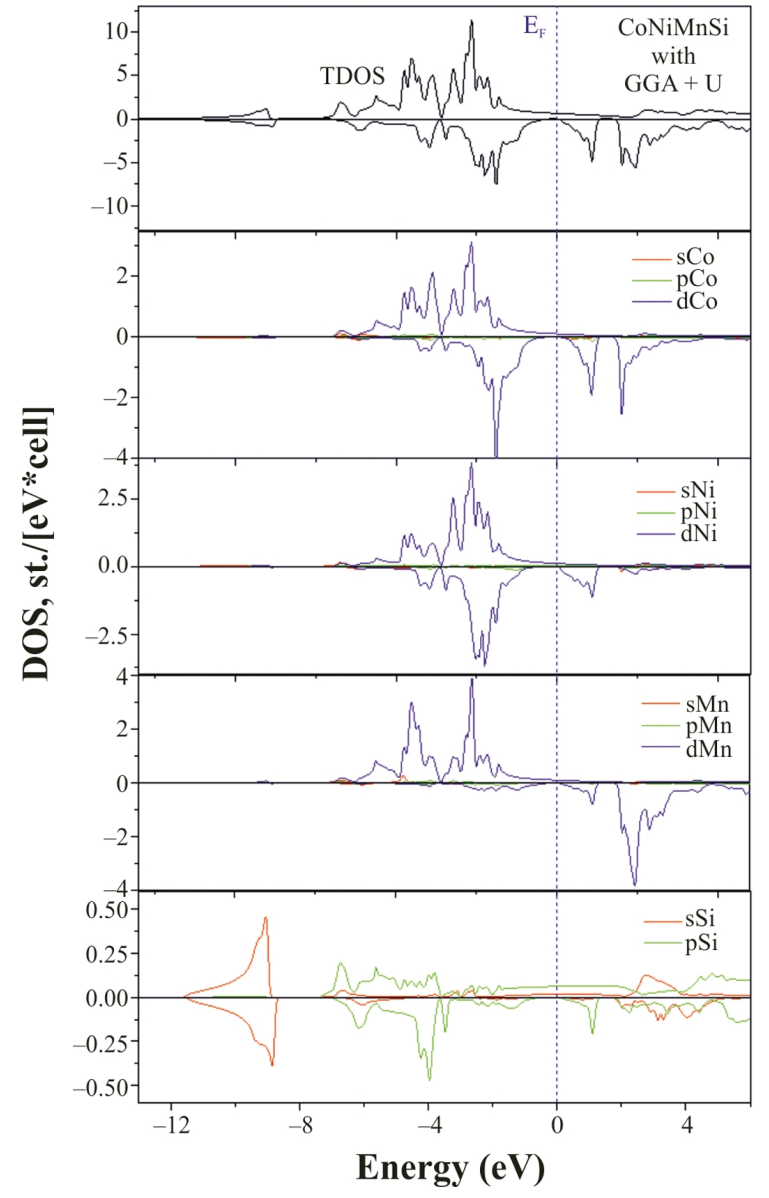

Fig. 4. Total and partial densities of states (TDOS, PDOS) for CoNiMnSi in its stable structure types calculated using the GGA $+\mathrm{U}$ approximation.

respectively. From the figures it can be observed that the charge contours are similar and that the near spherical charge distributions around the $\mathrm{Si}$ atom sites are negligible, indicating that the $\mathrm{Si}-\mathrm{Mn}$, $\mathrm{Si}-\mathrm{Ni}$ and $\mathrm{Si}-\mathrm{Co}$ bonding is expected to be ionic in character. However, between the transition metal elements, the $\mathrm{Co}$ and $\mathrm{Ni}$ atoms hybridize with an Mn atom for minority and majority spin, suggesting a covalent interaction between $\mathrm{Co}$ and $\mathrm{Ni}$ with $\mathrm{Mn}$ atoms. Therefore, the bonding character may be described as a mixture of covalent and ionic character.

We will now also report on the magnetic properties of the studied compound. For this purpose, we have evaluated the total and the local 

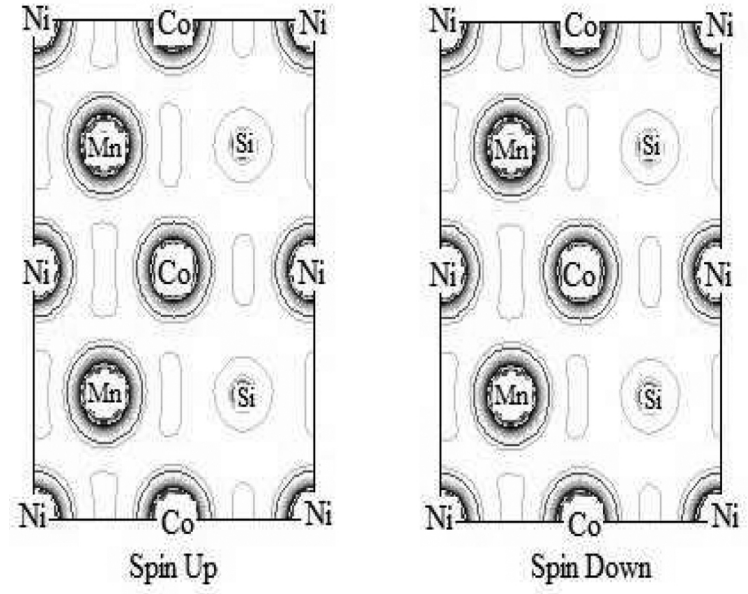

Fig. 5. Charge density distribution in the $\left(\begin{array}{lll}1 & 1 & 0\end{array}\right)$ plane of the CoNiMnSi quaternary Heusler compound calculated using the GGA approximation.

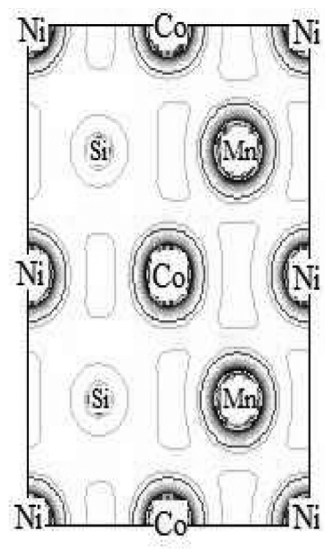

Spin Up

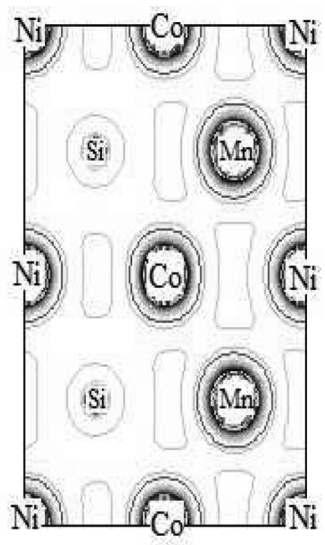

Spin Down
Fig. 6. Charge density distribution in the $\left(\begin{array}{lll}1 & 1 & 0\end{array}\right)$ plane of the CoNiMnSi quaternary Heusler compound calculated using the GGA + U approximation.

magnetic moments, which are presented in Table 4. We have found that the magnetic moment of the $\mathrm{Mn}$ atom is much higher than those of the other atomic constituents. We have also observed that the magnetic moments calculated with GGA + $\mathrm{U}$ are greater than those calculated with GGA as well as those previously obtained for the $\mathrm{Co}_{2} \mathrm{MnSi}$ compound [4, 25, 26, 29]. The local moment of the $\mathrm{Si}$ atom is negligibly small and aligned antiparallel to the $\mathrm{Co}, \mathrm{Ni}$ and $\mathrm{Mn}$ moments. It originates from the hybridization of the transition metals

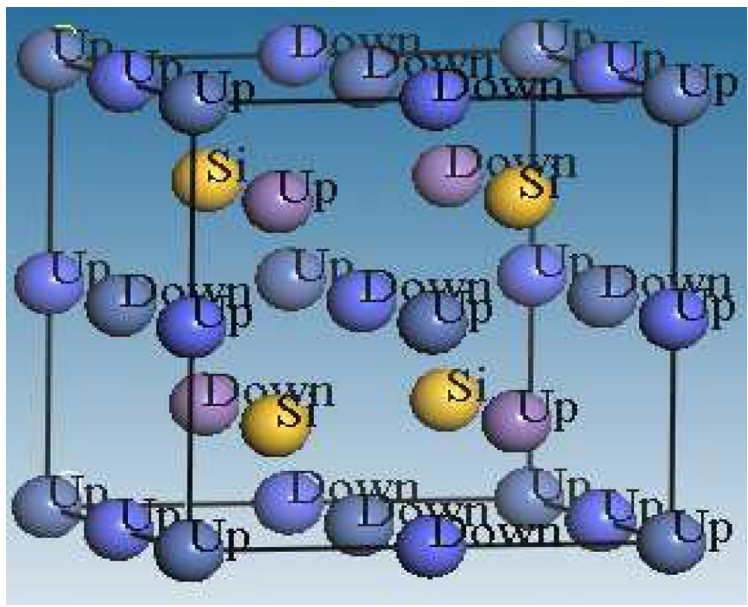

Fig. 7. The antiferromagnetic (AFM) configuration of CoNiMnSi.

and is caused by the overlap of the electron wave functions, as reported by Kandpal et al. [29]. In Table 5, we report the computed spin-polarization, magnetic exchange energy between ferromagnetic FM and anti-ferromagnetic AFM states $\left(\Delta \mathrm{E}_{(\mathrm{FM}-\mathrm{AFM})}\right)$ and Curie temperature $\left(\mathrm{T}_{\mathrm{c}}\right)$. The electron spin polarization $(\mathrm{P})$ at the Fermi energy $\left(\mathrm{E}_{\mathrm{F}}\right)$ of a material is defined by:

$$
P=\frac{\rho \uparrow\left(E_{F}\right)-\rho \downarrow\left(E_{F}\right)}{\rho \uparrow\left(E_{F}\right)+\rho \downarrow\left(E_{F}\right)}
$$

where $\rho \uparrow\left(\mathrm{E}_{\mathrm{F}}\right)$ and $\rho \downarrow\left(\mathrm{E}_{\mathrm{F}}\right)$ are the spin dependent densities of states at the Fermi level $\left(\mathrm{E}_{F}\right)$. The $\uparrow$ and $\downarrow$ symbols denote the majority and the minority states, respectively. We note from the results of the calculated electron spin polarization at $E_{F}$ that the $100 \%$ spin polarization characteristic of HM materials is observed in the studied compound when Coulomb interactions are considered. To find the ground state magnetic structure, we have performed total-energy calculations of different magnetic configurations, i.e. the ferromagnetic (FM) and anti-ferromagnetic (AFM) phases, to verify the magnetic stability of the investigated compound. The anti-ferromagnetic AFM configuration is shown in Fig. 7. By comparing the total energies, we find that the FM configuration for our compound is more stable than the AFM configuration. The calculated total energy difference of the FM state is lower than that of the AFM state 
Table 4. Total, interstitial and local magnetic moments.

\begin{tabular}{lccccccc}
\hline \multirow{2}{*}{ Compounds } & & $\begin{array}{c}\mu^{\text {Total }} \\
\left(\mu_{\mathrm{B}} / \text { Cell }\right)\end{array}$ & $\begin{array}{c}\mu^{\text {interstitial }} \\
\left(\mu_{\mathrm{B}} / \text { Cell }\right)\end{array}$ & \multicolumn{5}{c}{$\mu^{\text {atomic }}\left(\mu_{\mathrm{B}} /\right.$ Cell $)$} \\
& & & & $\mathrm{Co} 1$ & $\mathrm{Ni} / \mathrm{Co}_{2}$ & $\mathrm{Mn}$ & $\mathrm{Si}$ \\
\cline { 2 - 8 } $\mathrm{CoNiMnSi}$ & $\mathrm{GGA}$ & 4.69 & 0.038 & 1.036 & 0.458 & 3.18 & 0.0220 .038 \\
& $\mathrm{GGA}+\mathrm{U}$ & 5.99 & -0.03377 & 1.613 & 0.567 & 3.89 & \\
\hline \hline $\mathrm{Co}_{2} \mathrm{MnSi}$ & & $5.00^{\mathrm{a}, \mathrm{b}, \mathrm{c}, \mathrm{d}}$ & - & - & - & - & - \\
\hline${ }^{\mathrm{a}}[4],{ }^{\mathrm{b}}[25],{ }^{\mathrm{c}}[29],{ }^{\mathrm{d}}[26]$ & & & & & &
\end{tabular}

Table 5. Calculated spin-polarization, exchange energy $\left(\Delta \mathrm{E}_{(\mathrm{FM}-\mathrm{AFM}}\right)$, and Curie temperature $\left(\mathrm{T}_{\mathrm{c}}\right)$.

\begin{tabular}{cccc}
\hline Compounds & $\begin{array}{c}\Delta \mathrm{E}_{(\mathrm{FM}-\mathrm{AFM})} \\
(\mathrm{mRy} / \mathrm{Atom})\end{array}$ & $\mathrm{T}_{\mathrm{C}}(\mathrm{K})$ & Spin polarization ratio (\%) \\
\hline \hline CoNiMnSi & -0.011 & $871.89 \mathrm{GGA}$ & $56 \%$ GGA \\
& & $1107.19 \mathrm{GGA}+\mathrm{U}$ & $100 \% \mathrm{GGA}+\mathrm{U}$ \\
\hline $\mathrm{Co}_{2} \mathrm{MnSi}$ & & $928^{\mathrm{a}}$ & $100 \%$ \\
& & $985^{\mathrm{b}, \mathrm{c}}$ & \\
\hline
\end{tabular}

$\mathrm{a}[4],{ }^{\mathrm{b}}[27],{ }^{\mathrm{c}}[28]$

by about $-0.011046 \mathrm{mRy} /$ atom, as shown in Table 5. The Curie temperatures $\mathrm{T}_{\mathrm{c}}$ for CoNiMnSi were estimated according to the model presented in the literature $[4,39]$ by applying the linear relation $\mathrm{T}_{\mathrm{c}}=23+181 \mu^{\text {Total }}$, where $\mu^{\text {Total }}$ is the total magnetic moment. The values obtained are $871.89 \mathrm{~K}$ and $1107.19 \mathrm{~K}$ for the GGA and GGA $+\mathrm{U}$ approximations, respectively.

\section{Conclusions}

In this study, we performed first-principles calculations of the structural stability as well as the mechanical, electronic and magnetic properties for the quaternary Heusler alloy CoNiMnSi by employing the FP-LAPW method. The exchangecorrelation functional was evaluated within the GGA and GGA + U approximations. The ground state properties, including the lattice parameter and the bulk modulus for the $\mathrm{LiMgPbSb}, \mathrm{Hg}_{2} \mathrm{CuTi}$ and $\mathrm{Cu}_{2} \mathrm{MnAl}$-type phases have been determined. The CoNiMnSi compound was found to be stable in the $\mathrm{LiMgPbSb}$-type phase. The high values of the bulk modulus and Young's modulus revealed the strong incompressibility of this compound. The analysis of the electronic properties revealed that this compound exhibits a gap in the minority states band and is a half-metallic ferromagnet. From the total energy calculation, we can state that this compound has a ferromagnetic ground state with a high spin polarization. The magnetic moments of the studied compound were calculated to be $4.69 \mu_{\mathrm{B}}$ and $5.99 \mu_{\mathrm{B}}$ using the GGA and GGA $+\mathrm{U}$ approximations, respectively. The Curie temperature for this compound was also determined. Finally, we state that our study demonstrates that this compound is adequate for spintronic applications.

\section{Acknowledgements}

The authors (K.R. and S.B.O) acknowledge the financial support by the Deanship of Scientific Research at King Saud University for funding the work through the Research Group Project No. RPG-VPP-088.

\section{References}

[1] Groot R.A.D., Mueller F.M., Engen P.G.V., Buschow K.H.J., Phys. Rev. Lett., 50 (1983), 2024.

[2] Galanakis I., Dederichs G.A., PapaniKoLAOU N., J. Phys. D, 39 (2006), 765.

[3] Rai D.P., Shankar A., SANDeEP, Ghimire M.P., THAPA R.K., Physica B, 407 (2012), 3689.

[4] Candan A., UĞUr G., Charifi Z., BaAziz H., ElLiAltioĞLU M.R., J. Alloy. Compd., 560 (2013), 215.

[5] Drews J., Eberz U., SChuster H., J. Less-Common. Met., 116 (1986), 271. 
[6] Dai X., LiU G., Fecher G.H., Felser C., Li Y., LIU H., J. Appl. Phys., 105 (2009), 07E901.

[7] Al-Zyadi J.M.K., GaO G.Y., YAO K.L., J. Magn. Magn. Mater, 378 (2015), 1.

[8] Feng Y., Chen H., Yuan H., Zhou Y., Chen X., J. Magn. Magn. Mater, 378 (2015), 7.

[9] Berri S., Maouche D., Ibrir M., Zerarga F., J. Magn. Magn. Mater, 354 (2014), 65.

[10] Halder M., Mukadam M.D., Suresh K.G., Yusuf S.M., J. Magn. Magn. Mater., 377 (2015), 220.

[11] Gao G.Y., Hu L., Yao K.L., Luo B., LiU N., J. Alloy. Compd., 551 (2013), 539.

[12] Slater J.C., Adv. Quant. Chem., 1 (1964), 5564.

[13] Blaha P., Schwarz K., Sorantin P., Tricky S.B., Comput. Phys. Commun., 59 (1990), 399.

[14] Schwarz K., Blaha P., Madsen G.K.H., Comput. Phys. Commun., 147 (2002), 71.

[15] Wong K.M., Alay-E-Abbas S.M., Shaukat A., FANG Y., LEI Y., J. Appl. Phys., 113 (2013), 014304.

[16] Wong K.M., Alay-E-AbBas S.M., FAng Y., Shaukat A., Lei Y., J. Appl. Phys., 114 (2013), 034901.

[17] Blaha P., Schwarz K., Madsen G.K.H., KvasNICKA D., LUITZ J., WIEN2K, An Augmented Plane Wave + Local Orbitals Program for Calculating Crystal Properties, Wien, 2001.

[18] Perdew J.P., Burke S., Ernzerhof M., Phys. Rev. Lett., 77 (1996), 3865.

[19] Perdew J.P., Burke S., Wang Y., Phys. Rev. B, 54 (1996), 16533.

[20] Monkhorst H.J., PACK J.D., Phys. Rev. B, 13 (1976), 5188.

[21] Anisimov V.I., Aryasetiawan F., Liechtenstein A.I., J. Phys. Condens. Matter., 9 (1997), 767.

[22] Liechtenstein A.I., Anisimov V.I., ZAAnen J., Phys. Rev. B, 52 (1995), R5467.

[23] Loschen C., Carrasco J., Neyman K.M., IlLAS F., Phys. Rev. B, 75 (2007), 035115.

[24] Murnaghan F.D., Proc. Natl. Acad. Sci. USA, 50 (1944), 697.

[25] Picozzi S., Continenza A., Freeman A.J., Phys. Rev. B, 66 (2002), 094421.
[26] Gökoglu G., Gülseren O., Eur. Phys. J. B, 76 (2010), 321.

[27] Brown P.J., NeumanN K.U., Webster P.J., ZIEBECK K.R.A., J. Phys. Condens. Mater., 12 (2000), 1827.

[28] Raphael M.P., Ravel B., Willard M.A., Cheng S.F., Das B.N., Stroud R.M., Bussmann K.M., Claassen J.H., Harris V.G., Appl. Phys. Lett., 79 (2001), 4396.

[29] Kandpal H.C., Fecher G.H., Felser C., J. Phys. D, 40 (2007), 1507.

[30] Rached H., Rached D., Khenata R., ReshaK A.H., Rabah M., Phys. Status Solidi B, 246 (2009), 1580.

[31] Fedoras F.I., Theory of Elastic Waves in Crystals, Oxford University Press, New York, 1985.

[32] Born M., HuAng K., Dynamical Theory and Experiment I, Springer Verlag, Berlin, 1982.

[33] Rached H., Rached D., Rabah M., Khenata R., Reshak A.H., Physica B, 405 (2010), 3515.

[34] Voigt W., Lehrbuch der Kristallphysik, Springer, Berlin, 1928.

[35] Haines J., Leger J.M., Bocquillon G., Annu. Rev. Mater. Res., 31 (2001), 1.

[36] Anderson O.L., J. Phys. Chem. Solids, 24 (1963), 909.

[37] Schreiber E., Anderson O.L., Soga N., Elastic Constants and their Measurements, McGraw-Hill, NewYork, 1973.

[38] Biskri Z.E., RACHED H., BOUChEAR M., RACHED D., J. Mech. Behav. Biomed. Mater, 32 (2014), 345.

[39] Wurmehl S., Fecher G.H., Kandpal H.C., Ksenofontov V., Felser C., Lin H.J., Morais J., Phys. Rev. B, 72 (2005), 184434.
Received 2015-05-29

Accepted 2015-11-18 